\section{Inside or Outside: Detecting the Cellular Location of Bacterial Pathogens}

\author{
BioTechniques 30:304-311 (February 2001)
}

\begin{abstract}
Salmonella are intracellular pathogens that infect and multiply inside macrophages. Although Salmonella are some of the beststudied pathogens, it is difficult to determine quickly and reliably whether the bacteria are intracellular or extracellular. We have developed a novel method using differential fluorescence of two fluorescent proteins to determine the cellular location of pathogenic bacteria in macrophage infection assays. Using the differential expression of two unique fluorescent proteins that are expressed under specific conditions, we have developed a real-time assay for macrophage infections. The critical advantages of this system are that it does not alter the bacterial surface, it is not toxic to either the bacteria or the host cell, and it may be used in realtime quantitative assays. This assay can be readily applied to any other model pathogenic systems such as Listeria, Mycobacteria, and Legionella in which intracellular gene expression has been characterized.
\end{abstract}

\section{INTRODUCTION}

Salmonella are facultative intracellular parasites that can infect and multiply inside of macrophages. The virulence of Salmonella has been studied for many years, and intricate details are known about the mechanisms by which Salmonella infects macrophages (4). Most studies on Salmonella pathogenesis have relied on essentially three types of assay to determine the cellular location. A great deal has been gleaned from the first approach, direct observation by electron microscopy of cells fixed in various stages of infection (11). A second approach, the gentamicin protection (or exclusion) assay, has become the de facto standard assay for determining cellular location and relies on the inability of the antibiotic gen- tamicin to penetrate the eukaryotic cell membrane (3). This antibiotic rapidly kills the gentamicin-sensitive bacteria that remain extracellular, but bacteria inside the macrophage are resistant to the toxicity and survive. Aliquots not treated with gentamicin are plated to give the total number of bacteria in a sample, and aliquots treated with gentamicin are plated to give the number of intracellular bacteria (2). The third approach relies on fluorescence imaging techniques to count bacteria. Bacteria are labeled with a fluorophore such as fluorescein isothiocyanate (FITC) (which fluoresces green) before incubation with macrophages. After the incubation period, the macrophages are fixed and permeabilized, if necessary, and then stained with labeled antibodies or other dyes for visualization (10).

Each of these approaches has its drawbacks. All of these techniques are finite end-point assays that result in the destruction of the sample. Furthermore, the assay itself may interfere with the process of infection. For example, macrophages have specific receptors for FITC, so labeling bacteria with FITC before performing a macrophage assay may affect the uptake of the bacteria by the macrophage and affect the outcome of the assay (15). Similarly, visualization by electron microscopy or differential fluorescence microscopy traditionally requires that the sample be fixed. Gentamicin assays require a period of incubation of the antibiotic, the macrophages, and the bacteria. They then require time for the bacterial colonies to grow. In all three assays, the detection of infection when a low multiplicity of infection is used is extremely difficult.

We have circumvented the problems associated with these assays and developed a novel method for studying the interactions of pathogens with macrophages. Our assay has three essential elements: (i) the assay can be performed in real time without the need for staining or fixing; (ii) the assay does not alter the macrophage or bacterial cell surface and therefore should not interfere with the uptake process; and (iii) the assay is applicable to many systems.

The assay uses differential fluorescence using two fluorescently labeled proteins that are differentially expressed. Extracellular bacteria produce red fluorescence, and intracellular bacteria produce both green and red fluorescence. These two fluorescent signals can be used to detect macrophage infection by microscopy or flow cytometry.

\section{MATERIALS AND METHODS}

\section{Plasmids and Strains}

Salmonella enterica sv. Enteritidis ( $S$. Enteritidis) strain LK5 is wild-type, pathogenic, and prototrophic (6). Bacteria were routinely grown overnight at $37^{\circ} \mathrm{C}$, with aeration, in LB broth supplemented with either ampicillin (at a final concentration of $100 \mu \mathrm{g} / \mathrm{mL}$ ) or chloramphenicol (at a final concentration of $30 \mu \mathrm{g} / \mathrm{mL}$ ) when required (7). Plasmid pFM10.1 was a generous gift from Stanley Falkow, Stanford University. This plasmid contains a fusion between the promoter for $s$ s $a H$ and the green fluorescent protein (GFP), and the fusion was initially identified by fluorescenceactivated cell sorting to be expressed inside of the macrophages $(13,14)$. pFM10.1 is a derivative of pBR322 and uses the colE1 origin for replication.

pRE233 was constructed by digesting pDS-Red (Clontech Laboratories, Palo Alto, CA, USA) with $X b a \mathrm{I}$ and cloning the resulting 719-bp fragment

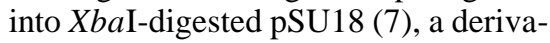
tive of pACYC184 that contains the $\alpha$ complementing fragment of lacZ. This cloning restores the appropriate reading frame of the lacZ-rfp fusion and, hence, the lac promoter on the plasmid controls the transcription of $r f p$. Colonies were screened for clones by their color; the red fluorescent protein (RFP) gives bacterial colonies a pink hue. pRE233 is a derivative of pACYC184 and uses the $\mathrm{p} 15 \mathrm{~A}$ origin for replication.

\section{Isolation of Peritoneal Cells}

Female BALB/c mice between six and eight weeks old were purchased from Harlan-Sprague (Indianapolis, IN, USA) and housed at the University of Illinois according to the local laboratory animal care advisory committee (LACAC) guidelines. Mice were sacrificed by $\mathrm{CO}_{2}$ asphyxiation, and follow ing brief sterilization in ethanol, the skin was removed, leaving the perito- 


\section{Short Technical Reports}

neum intact. Five milliliters of ice-cold tissue culture media (RPMI) were injected into the peritoneal cavity, and after approximately $1 \mathrm{~min}$, the tissue culture media were aspirated into a syringe. This was immediately placed on ice and kept cold until it was used.

\section{Fluorescence Microscopy}

Macrophages and fluorescent bacteria were quantified by flow cytometry and diluted to give an multiplicity of infection (MOI) of 1:1-5:1, as described below. The invasion assays were performed as described below. Following incubation with the macrophages, the samples were resuspended by mixing with a pipet tip, and $200-\mu \mathrm{L}$ samples were prepared by centrifugation onto a microscope slide using a Thermo Shandon Cytospin ${ }^{\circledR}$ II (Shandon, Pittsburgh, PA, USA). Samples were stained with Wright-Giemsa stain (Fisher Scientific, Pittsburgh, PA, USA) for light microscopy to characterize the peritoneal cells or were left unstained for fluorescence analysis. A $600 \times$ final magnification was used. The filters used to detect GFP fluorescence were $485( \pm 24) \mathrm{nm}$ for excitation and $537( \pm 40) \mathrm{nm}$ for emission. The filters used to detect RFP fluorescence were $582( \pm 20) \mathrm{nm}$ for excitation and $625( \pm 30) \mathrm{nm}$ for emission.

\section{Flow Cytometry}

Bacteria were diluted in $0.85 \% \mathrm{NaCl}$ to an appropriate concentration to give an MOI of $1: 1-5: 1$. To calculate the MOI, samples of fluorescent bacteria were counted using an XL404 flow cytometer (Beckman Coulter, Fullerton, CA, USA) with detection based on the fluorescence markers. Macrophages were counted on the same machine by detecting the forward scatter of the laser. The volume of bacterial culture added to the macrophages was adjusted to provide equal numbers of bacteria and macrophages. The bacteria were added to the peritoneal macrophage preparation in RPMI tissue culture media, and the mixture was performed as described below. Following incubation with the macrophages, samples were resuspended into the tissue culture media by mixing with a pipet tip and were analyzed by flow cytometry. Because of the small size of bacteria, free bacteria in the absence of macrophages were detected by fluorescence. Macrophages were detected by forward scatter. The sample was gated to eliminate larger particles, particularly neutrophils. Excitation of the GFP was at $488 \mathrm{~nm}$ and $100 \mathrm{~mW}$ using an argon ion laser, and emission was detected at $530 \mathrm{~nm}$ with a 40-nm bandpass filter (measuring 510-
$550 \mathrm{~nm})$. Excitation of the DS-Red was at $568 \mathrm{~nm}$ and $50 \mathrm{~mW}$ with a krypton/ argon ion laser, and emission was detected at $630 \mathrm{~nm}$ with a 40-nm bandpass filter (measuring 610-650 nm). Data were analyzed using Summit software (Cytomation, Fort Collins, CO, USA).

\section{Gentamicin Protection Assays}

These assays were performed as described elsewhere (3) with minor modifications. Fluorescent bacteria and peritoneal macrophages were both quantified by flow cytometry and diluted in saline or tissue culture media to a final MOI of approximately 1:1. Unlabeled bacteria were diluted in similar proportions before mixing with the macrophages. After $2 \mathrm{~h}$ of incubation at $37^{\circ} \mathrm{C}$ in $5 \% \mathrm{CO}_{2}$, samples were split into three aliquots. Gentamicin was added to a final concentration of 100 $\mu \mathrm{g} / \mathrm{mL}$ to one aliquot. The other two aliquots were not treated. After a further 2-h incubation at $37^{\circ} \mathrm{C}$ in $5 \% \mathrm{CO}_{2}$, one of the untreated aliquots was serially diluted in saline solution, plated on LBagar, and viable bacteria were counted after overnight growth. The other two aliquots were washed six times in icecold tissue culture media before dilution and plating for viable counts.

The untreated and unwashed sample

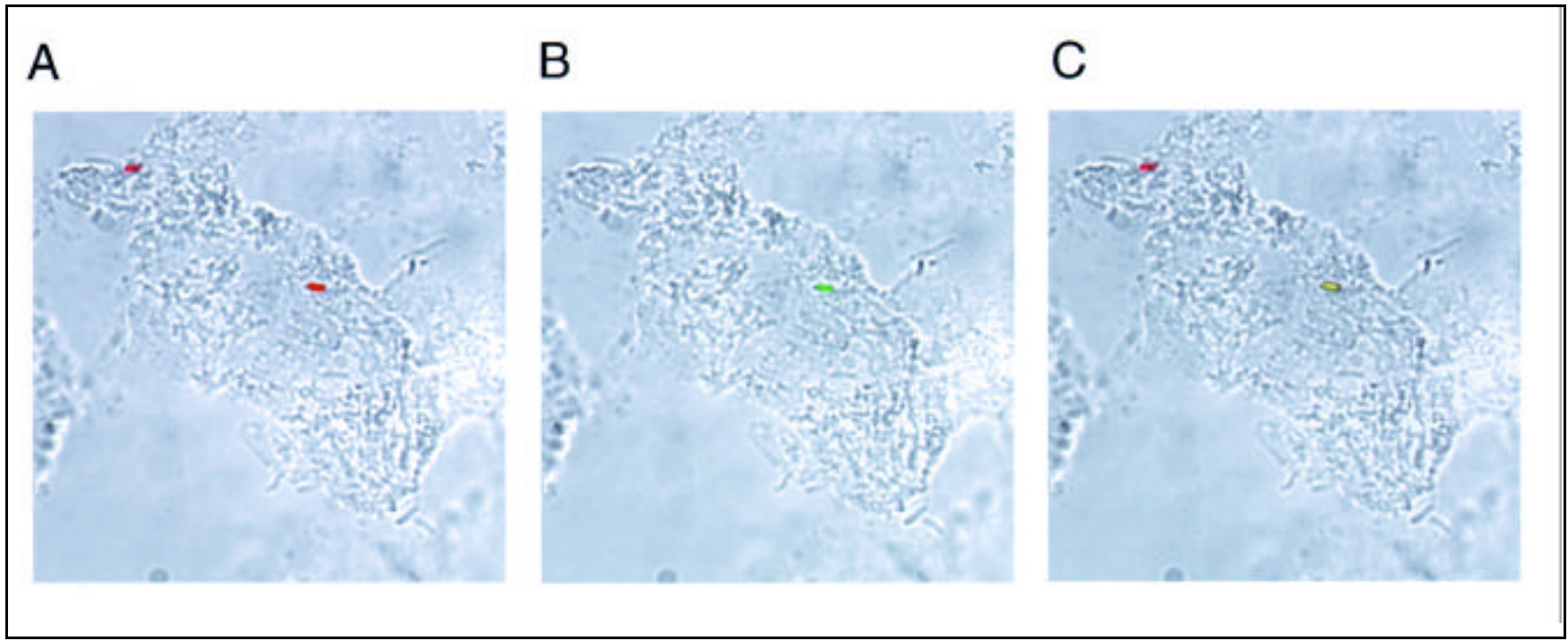

Figure 1. Infection of mouse peritoneal macrophages with $S$. Enteritidis viewed by fluorescence microscopy. After incubation of the bacteria and the macrophages, the samples were prepared by cytocentrifugation and then visualized by light and fluorescence microscopy. Two bacteria are present in this field. In panel A, both fluoresce red from of the RFP visualized using the Texas Red ${ }^{\circledR}$ filter set. In panel B, only the lower bacterium is green as it is intracellular (and hence expressing GFP), but the upper bacterium is extracellular. The GFP was detected using the FITC filter set. In panel C, an overlay of panels A and B, the intracellular bacterium appears yellow, but the extracellular bacterium appears red. 


\section{Short Technical Reports}

provided the total number of bacteria added to the macrophages. The untreated and washed sample provided the total number of adherent and intracellular bacteria. The gentamicin-treated and washed sample provided the number of intracellular bacteria.

\section{Calculations and Statistics}

Flow cytometry counts the number of macrophages that pass through the laser beam and scores whether the macrophage has fluorescent bacteria associated with it. In contrast, gentamicin protection assays score the number of viable bacteria and does not count the number of macrophages. Therefore, to effectively compare the gentamicin protection assay with the flow cytometry data, we assume that each macrophage has only a single bacteria associated with it. Before the infections, macrophages and fluorescent bacteria were counted by flow cytometry, and the ratio was adjusted to give an MOI between $0.8: 1$ and 1.2:1. Even though the gentamicin protection assays were performed with such low MOI, the population will have a Poisson distribution in which some macrophages will have several bacteria associated with them and some will have none. Hence, we assume that the bacterial viable count approximates to the number of macrophages with adherent or internalized bacteria. Therefore, to calculate the percentage of macrophages that have bacteria associated with them (Figure 3), the bacterial viable count is divided by the total number of macrophages. The variance between the numbers of macrophages with bacteria attached or internalized was analyzed using two-way ANOVA, and the difference between the means was analyzed using the student's $t$-test.

\section{RESULTS AND DISCUSSION}

\section{Rationale for the Dual-Color Labeling}

Since the cloning and characterization of $g f p$, which encodes the GFP from Aequorea victoria, several mutations in $g f p$ were identified that altered the spectral properties of the resulting protein, potentially allowing dual-color labeling. However, all of these clones are point mutations in $g f p$, and because the wild-type and the mutant derivatives can recombine, they can not both be maintained on plasmids in a single cell. In contrast, the DS-Red $r f p$ gene from
Discosoma sp., which encodes the red fluorescent protein, has no homology with $g f p$ and therefore allows the stable maintenance of both $g f p$ and $r f p$ in a single bacterial cell. We cloned the $r f p$ gene onto pSU18, a derivative of pACYC184, which has a compatible origin of replication with pBR322. Both plasmids can be stably maintained in Salmonella.

Expression of $r f p$ is controlled by the lac promoter, which in the absence of LacI is constitutively expressed. (Salmonella are naturally $l a c^{-}$and therefore do not contain lacI.) Expression of $g f p$ from the $\mathrm{pBR} 322$ derivative is controlled by the promoter for $s \mathrm{saH}$, a gene that has been shown to be required for Salmonella virulence and whose expression is specifically induced when Salmonella invade macrophages $(13,14)$. The $g f p$ expression is induced, the protein is expressed and folded, and GFP can be detected within 15 min of the bacteria entering the macrophage. Furthermore, there appear to be no detrimental affects of GFP expression on Salmonella infection of macrophages $(1,12)$. This com bination of GFP and RFP results in bacteria that are always fluorescing red and specifically induce green fluorescence when they enter macrophages.
A

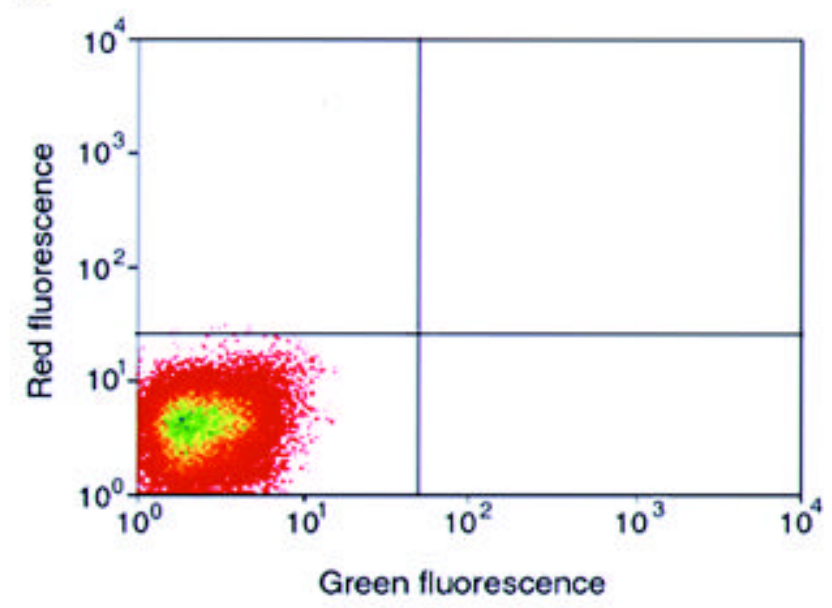

B

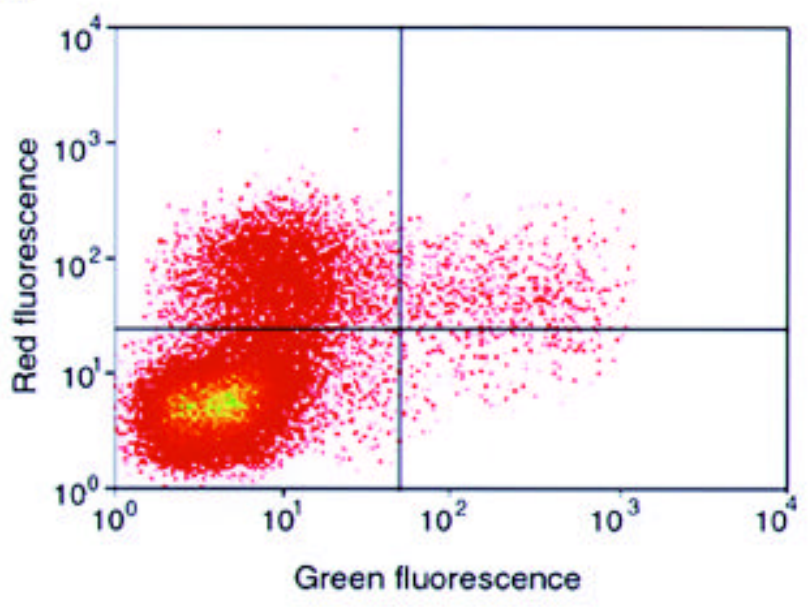

Figure 2. Infection of mouse peritoneal macrophages with $\boldsymbol{S}$. Enteritidis quantitated by flow cytometry. The abscissa represents the amount of green fluorescence on an arbitrary logarithmic scale, and the ordinate represents the amount of red fluorescence on an arbitrary logarithmic scale. The artificially colored image represents the number of events at each point with the scale increasing from red to green. Uninfected macrophages (A) have basal levels of both red and green fluorescence. When macrophages were infected with bacteria, two sub-populations emerged, those with increased red fluorescence- notice the increased events in the upper left quadrant —and those with increased red and green fluorescence — notice the increase in events in the upper right quadrant. 


\section{Fluorescence Microscopy}

To test the dual-color labeling, we infected peritoneal cells with $S$. Enteritidis at an initial MOI between 1:1 and 5:1. Figure 1 shows two bacteria associated with a single macrophage. Both bacteria fluoresce red (panel A) because of expression of $r f p$; however, only one of the bacteria fluoresces green (panel B), indicating that one of the bacteria (the red one) is extracellular, while the other bacteria (the red and green one) is intracellular. In an overlay of the two-color image, intracellular bacteria are yellow and extracellular bacteria are red.

\section{Flow Cytometry}

The cellular location of bacteria was also determined and quantitated by flow cytometry. To initially characterize the emissions, samples were quantified by detecting particles based on their side scatter. The emission of both green and red fluorescence was measured from either free bacteria or free macrophages (data not shown). Bacteria labeled only with RFP had a slightly increased fluorescence on the green channel compared to background (unlabeled) levels; however, this was at least two logs less intense than the fluorescence from GFP on this channel. There was no detectable increase in the red channel from GFP-labeled bacteria. This ensured that both the GFPs and RFPs could be quantitated separately whether the bacteria expressed GFP, RFP, or both. Under these conditions, mouse peritoneal macrophages emit minimal levels of red and green fluorescence (Figure 2A), which are significantly less than bacteria labeled with either of the fluorescent proteins.

Following incubation of bacteria and macrophages, three populations of cells are detected (Figure 2B). Most macrophages in this sample are not labeled and have background levels of fluores-

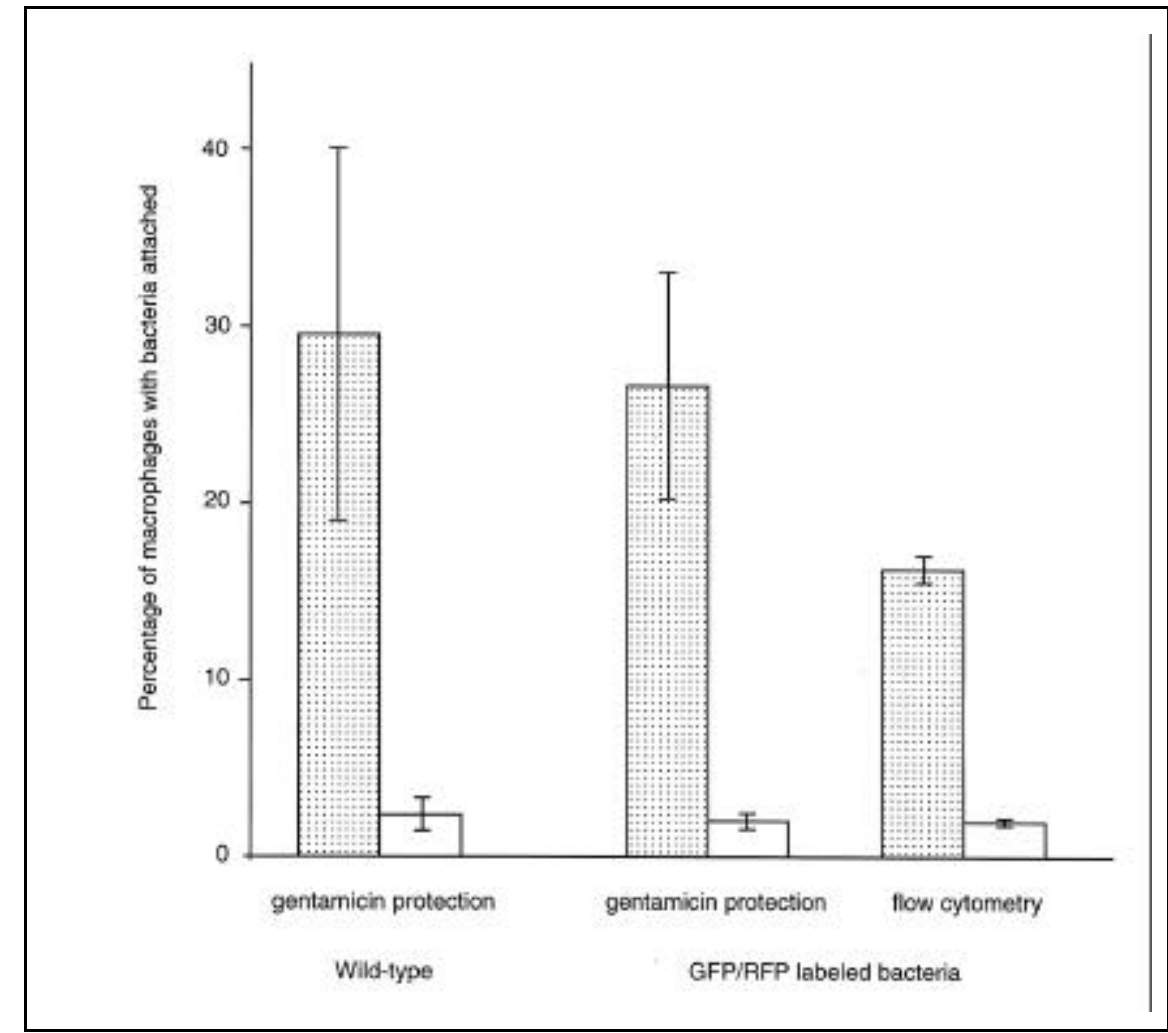

Figure 3. Attachment to and invasion in macrophages quantitated by gentamicin protection assays and flow cytometry. The stippled bars represent the number of macrophages that have bacteria attached, and the open bars represent macrophages with internalized bacteria. Infection of wild-type and RFP/GFP-labeled bacteria were compared by gentamicin protection assays. Infections of RFP/GFP-labeled bacteria were compared by gentamicin protection and flow cytometry. Data were analyzed by twoway ANOVA. These data are the average of four independent infections for each sample. 
cence. This level is identical to the level found in Figure 2A. A smaller sub-population, accounting for $15 \%-20 \%$ of the macrophages, have only red fluorescence, suggesting that these are macrophages with bacteria attached to the surface, but the bacteria have not invaded the macrophage. The third sub-population, accounting for approximately $2 \%$ of the total macrophages, has both red and green fluorescence, indicating that the bacteria have successfully invaded these macrophages, inducing GFP expression. As expected, very few particles were detected that had green fluorescence but not red fluorescence, and this population probably reflects the normal variance in red fluorescence.

\section{Gentamicin Protection Assays}

The novel two-color flow cytometric assay provides a rapid and sensitive method of assaying bacterial invasion into peritoneal cells. To validate this novel approach, we compared the attachment and invasion of $S$. Enteritidis to peritoneal macrophages using gentamicin protection assays and flow cytometry (Figure 3 ). We did not opsonize the bacteria before adding them to the macrophages, nor did we centrifuge the bacteria onto the macrophages, and we used a low MOI (1:1). These three experimental conditions do not result in close to $100 \%$ invasion of the macrophages by Salmonella, which can be achieved under optimal conditions (3). However, this protocol was efficacious for discriminating between attachment and invasion- $-20 \%-30 \%$ of the macrophages had bacteria attached to their surface but only $2 \%-5 \%$ of the macrophages had internalized bacteria.

When wild-type bacteria are compared with fluorescently labeled bacteria, there is no statistically significant difference in the attachment of bacteria to macrophages (from student's $t$-test: $P=0.64)$. Similarly, there is no statistically significant difference in the invasion into macrophages between these two bacterial populations (from student's $t$-test: $P=0.30$ ). This suggests that there is no effect of the GFP and RFP proteins on either attachment or internalization.

When attachment is quantitated by either flow cytometry or gentamicin protection assays, there is a statistically significant difference between the mean numbers of bacteria attached to each macrophage, depending on the quantitation used (from a students $t$ test: $P=0.02)$. Less attached bacteria are identified by flow cytometry (Figure 3 ). In contrast, when invasion is quantitated by the same methods, there is a much less statistically significant difference in the mean number of macrophages with internalized bacteria (from a students $t$-test: $P=0.14$ ). This suggests that either the flow cytometry is slightly under-representing the number of macrophages with attached bacteria or the gentamicin protection assay is over-representing this number. In either case, the error is probably introduced by more than one bacterium being attached to a single macrophage. For example, if a single macrophage has two bacteria attached to the surface, the flow cytometric analysis will only count this as a single event. However, the viable count assay will identify this as two bacteria but will not identify how many macrophages to which they were attached.

The number of bacteria attached to each macrophage is defined by the Poisson distribution, based on the number of macrophages and the number of bacteria. It is therefore possible to correct for the sampling error by reducing the ratio of infection below $1: 1$, thus altering the distribution of macrophages and bacteria. This will ensure that the majority of macrophages have 0 or 1 bacteria attached and only a very small minority will have more than one bacterium attached. Experiments using gentamicin protection assays routinely use an MOI between 10:1 and 50:1 to ensure the high levels of infection of the macrophages needed for statistically significant data analysis. In contrast, flow cytometric techniques allow rapid sampling of large volumes and dilute solutions, and, therefore, this high level of infection is not necessary for statistical significance.

\section{Summary}

In this paper, we demonstrated the application of dual-color fluorescence to study macrophage infections in Salmonella, but this approach may be easi- ly applied to any species for which intracellular gene expression has been characterized. This is an ever-expanding list of pathogens that currently includes Mycobacteria $(8,14)$, Legionella (9), Listeria (5), and the enteric pathogens like Salmonella and Yersinia (14). The simple construction of two plasmids, one with an inducible GFP and the second with a constitutive secondcolor fluorescence (such as the RFP), will allow rapid, real-time quantitation of bacterial invasion.

This assay also has an advantage over traditional invasive assays in that exquisite levels of quantitation can be achieved by flow cytometry. By counting large populations of macrophages and bacteria, rare events can rapidly garner statistical significance. Flow cytometry also allows quantitation of assays performed at very low MOI, which may better reflect a natural situation than overloading the macrophages with bacteria. Furthermore, using fluorescent proteins in this assay system allows gentamicin to be used in alternative ways, for example, as a selective marker for transposon insertions.

\section{REFERENCES}

1.Cormack, B.P., R.H. Valdivia, and S. Falkow. 1996. FACS-optimized mutants of the green fluorescent protein (GFP). Gene 173:33-38.

2.Edwards, R.A., D.M. Schifferli, and S.R. Maloy. 2000. A role for Salmonella fimbriae in intraperitoneal infections. Proc. Natl. Acad. Sci. USA 97:1258-1262.

3.Elsinghorst, E.A. 1994. Measurement of invasion by gentamicin resistance. Methods Enzymol. 236:405-420.

4.Falkow, S. 2000. Living in stools is not as dumb as you think. J. Bacteriol. 182:33193322.

5.Freitag, N.E. and K.E. Jacobs. 1999. Examination of Listeria monocytogenes intracellular gene expression by using the green fluorescent protein of Aequorea victoria. Infect. Im mun. 67:1844-1852.

6.Keller, L.H., C.E. Benson, V. Garcia, E. Nocks, P. Battenfelder, and R.J. Eckroade. 1993. Monoclonal antibody-based detection system for Salmonella enteritidis. Avian Dis. 37:501-507.

7.Maloy, S.R., V.J. Stewart, and R.K. Taylor. 1996. Genetic Analysis of Pathogenic Bacteria. CSH Laboratory Press, Cold Spring Harbor, NY.

8.Plum, G. and J.E. Clark-Curtiss. 1994. Induction of Mycobacterium aviumgene expression following phagocytosis by human macrophages. Infect. Immun. 62:476-483. 
9.Rankin, S. and R.R. Isberg. 1994. Identification of Legionella pneumophila promopoters regulated by the macrophage intracellular environment. Infect. Agents Dis. 2:269-271.

10.Rest, R.F. 1995. Association of bacteria with human phagocytes. Methods Enzymol. 253:12-26.

11.Takeuchi, A. 1967. Electron microscope studies of experimental Salmonella infection. I. Penetration into the intestinal epithelium by Salmonella typhimurium Am. J. Pathol. 50:109-136.

12. Valdivia, R.H. and S. Falkow. 1996. Bacterial genetics by flow cytometry: rapid isolation of Salmonella typhimurium acid-inducible promoters by differential fluorescence induction. Mol. Microbiol. 22:367-378.

13. Valdivia, R.H. and S. Falkow. 1997. Fluorescence-based isolation of bacterial genes expressed within host cells. Science 277:20072011.

14.Valdivia, R.H., A.E. Hromockyj, D. Monack, L. Ramakrishnan, and S. Falkow. 1996. Applications for green fluorescent protein (GFP) in the study of host-pathogen interactions. Gene 173:47-52.

15.Weingart, C.L., G. Broitman-Maduro, G. Dean, S. Newman, M. Peppler, and A.A. Weiss. 1999. Fluorescent labels influence phagocytosis of Bordetella pertussis by human neutrophils. Infect. Immun. 67:4264 4267.

We thank Anthea Lee and Stanley Falkow for the plasmids expressing GFP, Anne Nye for help with the fluorescence microscopy, and Gary Durack for help with the flow cytometry. This work was supported by National Institutes of Health grant no. PHS GM34715 and a grant from the Illinois Council for Food and Agriculture Research. Address correspondence to Dr. Robert A. Edwards, Department of Molecular Sciences, University of Tennessee, Memphis, Memphis, TN 38163, USA. e-mail: redwards@utmem.edu

Received 22 February 2000; accepted 21 September 2000.

\section{Robert A. Edwards and Stanley R. Maloy University of Illinois at Urbana-Champaign Urbana, IL, USA}

\section{Protein Aggregation Mediated by Cysteine Oxidation During the Stacking Phase of Discontinuous Buffer SDS-PAGE}

BioTechniques 30:311-316 (February 2001)

\begin{abstract}
The resolution of complex protein mixtures by discontinuous buffer SDS-PAGE is accomplished by their concentration into thin bands in the stacking gel, followed by their separation during migration through the resolving gel. Recombinant human interferon-inducible protein-10 (IP-10), a 10$k D a C-X$-C chemokine with four cysteines, aggregated during the stacking phase of $S D S-P A G E$ and generated $a$ band with an apparent molecular mass of $18 \mathrm{kDa}$. This aggregation depended on the presence of reduced sulfhydryl residues on IP-10, on the amount of loaded protein, and on the concentration of the ammonium persulfate used to polymerize the stacking gel. The aggregation of IP-10 could be prevented by reduction of its sulfhydryls with dithiothreitol followed by irreversible blockade with iodoacetamide. These methods may be useful in the prevention of aggregation of sulfhydryl-containing proteins during SDS$P A G E$, especially when large quantities are analyzed to assess their purity.
\end{abstract}

\section{INTRODUCTION}

The pioneering work of Laemmli (11) has made discontinuous buffer SDS-PAGE indispensable for protein analysis. The superior resolution of discontinuous buffer SDS-PAGE depends on the concentration of proteins in sharp bands away from the bulk of the SDS in the stacking gel, followed by their resolution during migration through the separating gel. The introduction of a discontinuous buffer system with Tricine (18) has further extended the range of resolution SDS-PAGE, by improving the separation of proteins smaller than $20 \mathrm{kDa}$. 